\title{
The Master's program in Advanced Optical Technologies: an interdisciplinary, international and individual approach
}

Jürgen Großmann, Bernhard Schmauss

Jürgen Großmann, Bernhard Schmauss, "The Master's program in Advanced Optical Technologies: an interdisciplinary, international and individual approach," Proc. SPIE 10452, 14th Conference on Education and Training in Optics and Photonics: ETOP 2017, 1045206 (16 August 2017); doi: 10.1117/12.2266243

Event: 14th Conference on Education and Training in Optics and Photonics, ETOP 2017, 2017, Hangzhou, China 


\title{
The Master's Program in Advanced Optical Technologies - an interdisciplinary, international and individual approach
}

\author{
Jürgen Großmann ${ }^{\mathrm{a}}$, Bernhard Schmauss ${ }^{\mathrm{a}}$ \\ ${ }^{a}$ Friedrich-Alexander Universität Erlangen-Nürnberg, Master's Program in Advanced Optical \\ Technologies, Paul-Gordan-Str. 6, 91052 Erlangen, Germany
}

\begin{abstract}
The Master's Program in Advanced Optical Technologies (MAOT) was established at the Friedrich-Alexander Universität Erlangen-Nürnberg in 2007 as part of the Elite Network of Bavaria (ENB), an initiative by the Bavarian State Government comprising about 40 elite Master's programs and doctoral programs. MAOT can be studied after a Bachelor in physics or an engineering subject. The Master's program realizes an innovative concept combining three core elements: (1) Interdisciplinarity: The program integrates courses and researchers from five engineering subjects and from physics. The degree of interdisciplinarity goes far beyond traditional programs. (2) Internationality: The program is taught entirely in English and special support is given to international students. (3). Individuality: The course curriculum was adapted at several points based on the experience in the initial years. The same is true for the way in which international students are supported and the type of support they need. The students are given an unusually high degree of freedom to develop an individual curriculum and to pursue research projects. Crucial experience and lessons learned are: (1) Lecturers and researchers have to be coordinated and the perspectives of the different disciplines have to be integrated within one program. Students must be guided in order to deal with the demands and challenges of the different disciplines. (2) International students need support with settling in Germany and with learning and working in a German cultural environment. They need support with administrative issues. Furthermore, they need to analyze and understand cultural differences and how they impact on the cooperation between lecturers and students and on the work in research groups. (3) Students must be helped to develop their own curriculum. They must learn how to combine their first-degree qualification with the specialized qualification which they gain after completing their Master's program. They need to develop the skills to match their preferences with what is realistic and feasible.
\end{abstract}

Keywords: optics, optical technologies, photonics, Master's program, curriculum development

\section{The framework}

The Master's Program in Advanced Optical Technologies (MAOT) at the Friedrich-Alexander Universität ErlangenNürnberg (FAU), Germany, was established within the framework of the Elite Network of Bavaria (ENB) in October 2007[1]. The ENB is an initiative by the Government of Bavaria, a state of the Federal Republic of Germany. It comprises several programs to support highly gifted student at universities (from bachelor to postdoctoral level).

MAOT is one of the Master's courses in the ENB that are intended to combine the support of highly gifted student with the development of innovative study concepts. At this time, there are 27 Master's courses from various disciplines (natural sciences, social sciences, engineering) within the ENB: 10 of these are currently funded by the ENB and 17 are continued programs that have exceeded the maximum funding duration of 10 years. There are also 18 doctoral programs and six Junior Research Groups at postdoctoral level.

\section{The German background}

\section{The MAOT program}

In Germany, Master's programs are almost always established as stand-alone courses and not as part of an integrated course with a doctoral program. They closely combine teaching with research. The usual duration is two years and admission required a Bachelor's degree of at least 3 years duration. The Bachelor's/ Master's system started to be used widely only about ten years ago as a result of the Bologna process. It replaced the Diplom system in which students studied for about 4.5 to 5 years to gain their first university degree before they could commence a doctorate. In this tradition, many people still regard the Master's degree as the first 'real' university degree, which replaces the former Diplom degree. Master's students are, therefore, rather regarded as more experienced undergraduates than as students on a postgraduate program.

14th Conference on Education and Training in Optics and Photonics: ETOP 2017, edited by Xu Liu,

Xi-Cheng Zhang, Proc. of SPIE Vol. 10452, 1045206 • ( 2017 ICO, IEEE, OSA, SPIE

CCC code: $0277-786 X / 17 / \$ 18 \cdot$ doi: $10.1117 / 12.2266243$ 


\section{The motivation}

Optical technologies are considered to be key enablers of future innovations. Moreover, it is a field characterized by rapid change and groundbreaking developments. There also tends to be significantly more fundamental research undertaken than in longer established technology fields, which demands a higher level of education and a more scientific approach [2]. However, not many degree programs in Germany (compared with the international situation) focus specifically on optics and optical technologies as yet; these subjects are generally included in a range of programs in science and engineering.

\section{The program}

MAOT is an international degree program taught exclusively in English. It is a four-semester program divided into three phases (figure 1):

- First semester: fundamental courses on optics, laser and numerical methods (25 ECTS $\left.{ }^{1}\right)$

- Second/ third semester: specialization courses in two of seven possible majors (Optical Metrology, Optics in Communication, Optical Materials and Systems, Optical Material Processing, Optics in Medicine, Computational Optics, Physics of Light) (nine courses with 45 ECTS in total)

- Fourth semester: Master's thesis (30 ECTS). Work on the Master's project only starts in the last phase of the study program (the actual average study time is five semesters). The subject of the thesis is not assigned earlier.

In addition students have to:

- conduct a research project of about 300 hours (10 ECTS)

- complete an internship outside the university (5 weeks, 5 ECTS)

- attend two lab courses in their majors (5 ECTS in total)

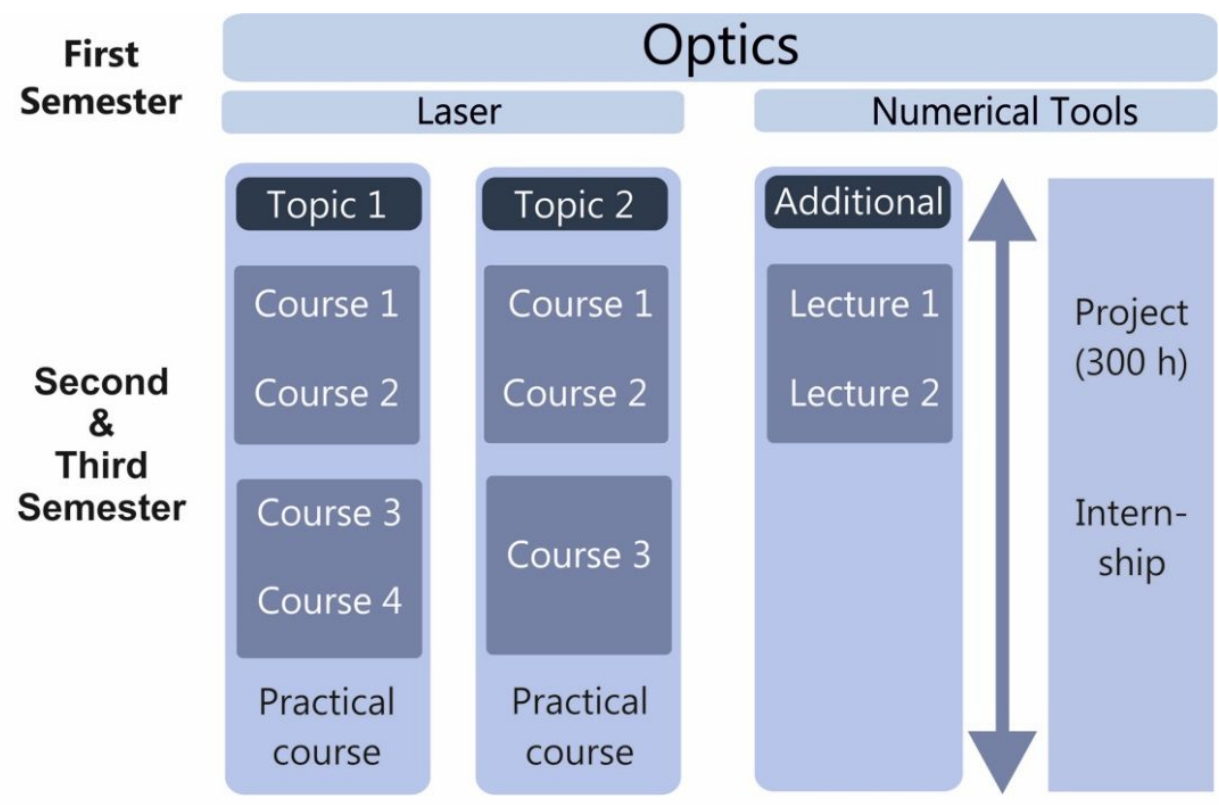

\section{$\begin{array}{ll}\text { Fourth } & \text { Master's Thesis }(900 \mathrm{~h})\end{array}$}

Figure 1: Structure of MAOT

\footnotetext{
${ }^{1}$ ECTS $=$ credit points according to the European Credit Transfer and Accumulation System, usually abbreviated to ECTS. One ECTS point corresponds to a workload of 30 hours.
} 
Students require a Bachelor degree in engineering or physics to be eligible to apply to MAOT. Since MAOT is part of the Elite Network of Bavaria, the demands are particularly high in terms of profile of the previous university, degree course, and grades achieved. Each year, 30-35 of around 180 applicants are admitted to MAOT. Of these, about 15-20 students actually start the program.

As can be seen from the structure of MAOT, the program aims to combine teaching on selected fields of application for optical technologies with knowledge of the fundamental physics behind these technologies. The choice of application fields was made based on how strongly the fields are represented in current research activities at FAU.

MAOT is embedded in a cluster of competence for optics and optical technologies in Erlangen together with the Max Planck Institute for the Science of Light (MPL) and the Graduate School in Advanced Optical Technologies (SAOT) as part of the German Excellence Initiative. On a daily basis, MAOT works in close cooperation with the SAOT doctoral program (joint courses, workshops and academies, sharing of technical and administrative infrastructure).

MAOT can be characterized by three core elements (figure 2):

- interdisciplinary

- international

- individual

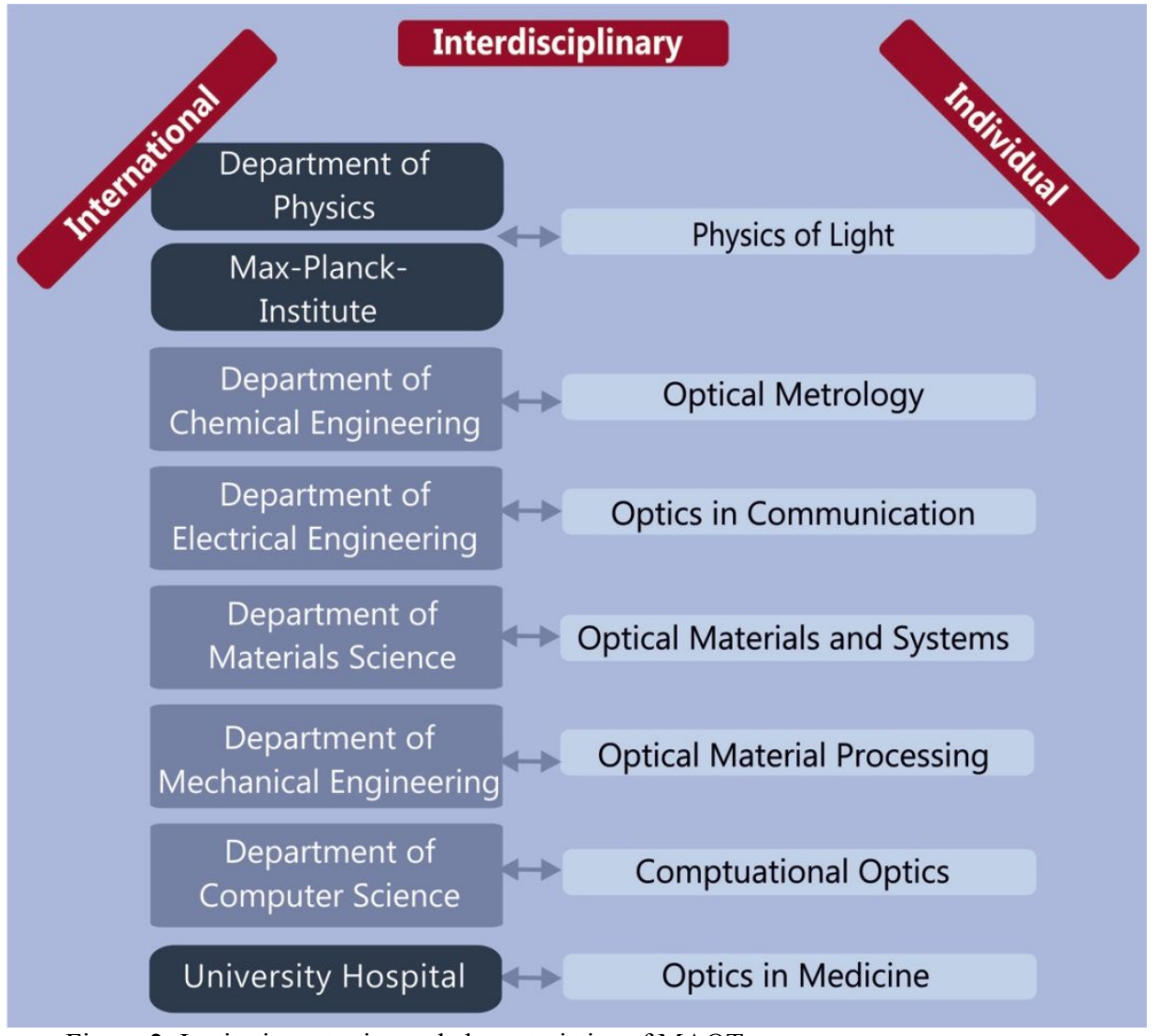

Figure 2: Institutions, topics and characteristics of MAOT 


\section{Interdisciplinary}

Essentially MAOT is an engineering program located at the faculty of engineering. It focuses on applications and prepares students for respective positions in industry or research institutes. For the major 'Optics in Medicine', the medical faculty and the university hospital take part in the program and contribute to teaching.

More important to the strong interdisciplinary approach, however, is the broad involvement of the physics department at the faculty of science. Due to rapid developments in the field of optical technologies and research topics often based on fundamental science, MAOT students need to understand the basics of physics more deeply than students of other engineering subjects. Therefore, MAOT offers comprehensive lectures on the fundamentals of optics and lasers, which go far beyond what is usually taught on engineering courses.

The physics department also contributes significantly to the specialization courses in the second/ third semesters. These deal mainly with current fields of research at the physics department or the Max Planck Institute. Students can also attend additional lectures in the fundamentals of optics if they choose the 'Physics of Light' major.

Moreover, MAOT maintains an extremely interdisciplinary collaboration within the faculty of engineering. All five departments of the faculty are represented through courses that are mainly but not exclusively linked to one of the major topics:

- Electrical engineering (Optics in Communication)

- Mechanical engineering (Optical Material Processing)

- Chemical engineering (Optical Metrology)

- Material science (Optical Materials)

- Computer science (Computational Optics)

Collaboration between MAOT (and also the SAOT doctoral program) and the engineering disciplines goes much further than in other combined degree programs such as mechatronics or in research projects.

\section{International}

For several reasons, MAOT was devised as an international program taught in English:

- The Elite Network of Bavaria wanted to attract highly gifted international students to Bavaria.

- MAOT is a research-oriented program and English is the universal scientific language.

- The interdisciplinary approach can be understood as a general emphasis on collaboration as the foundation of human work and life together. The training in international collaboration - a natural part of an international program - is one more aspect of this approach, which adds value to the MAOT qualification.

On average, around $25 \%$ of the students are from Germany each year; the rest come from 30 different countries (in particular: Iran, China, Ethiopia, and Pakistan). All courses are held in English.

International students are encouraged to learn German and language courses are offered exclusively for MAOT students. The majority of international students leave MAOT with A2 level German language skills; levels achieve range from A1 to $\mathrm{B} 2$.

\section{Individual}

MAOT enables students to choose a highly individual curriculum. The courses in the first semester are fixed, but the rest of the curriculum gives a high degree of freedom to the students. They can choose from seven possible majors and within each major they pick 3-4 of 5-6 courses. In addition, they can attend two courses which they are free to select from any subject area or from all courses at FAU as long as the course is related to MAOT topics.

They are also very free to choose a subject for the project report or the Master's thesis. These are not assigned to students but discussed with potential supervisors and usually a student can pick one of several offers.

Finally, students join MAOT from very different backgrounds. Typically, they will have a Bachelor's in one of four subject areas (fairly equally distributed): physics, optical engineering, electrical engineering, or another engineering 
field. The students have to adapt their very variable levels of knowledge and skills individually to the new subject matter taught at MAOT. This means that they have to link new material to existing knowledge. In several cases, they have to make up for gaps in material required for MAOT courses that was not covered in their Bachelor's degree programs. When possible, lecturers fill these gaps during courses, but cannot do so in all cases due to a lack of time and the very heterogeneous group of students.

\section{Outcome}

Since 2007, 113 students have completed MAOT, only $10 \%$ of them without graduating. The graduates can be divided into three groups:

- about $40 \%$ find a position in industry

- about 30\% do a doctorate in Erlangen (at an institute of the university or in collaboration with the Max Planck Institute)

- about $30 \%$ do a doctorate at another university in Germany or abroad (ratio approx. $60: 40$ )

Only a very small number do not continue their career in the field of optics or optical technologies.

\section{Developments}

During the ten years of MAOT there have been some vital developments, mainly within the first five years:

Course schedule of first semester. Form the very beginning, the plan was to offer courses in fundamentals in the first semester on which the specialization course in the second/ third semesters would build. However, initially these courses

- were devised with less focus on fundamentals than they are today (as it was assumed that all students start MAOT with some common knowledge of optics);

- included different courses for students with a background in physics or engineering;

- covered the subjects of the majors to a larger extent.

Several changes to this concept led to today's situation of two very comprehensive courses on the fundamentals of optics and lasers, a short introduction to numerical methods, and the possible majors. Due to the very mixed prior Bachelor's degrees and, even more so, the very heterogeneous curriculums in the various countries of origin (even for the same degree subject) it was necessary to offer more in-depth teaching in fundamentals to ensure a solid common foundation for the specialization courses. Furthermore, the students did not feel comfortable about being divided into different courses depending on the prior subject during the first semester as they felt insecure about the contents covered in the course that they had not attend.

Balancing specialization with broad qualification and freedom. Initially, students chose three major topics from six options (a seventh one - Physics of Light - was introduced later) each with three 5-ECTS courses. In addition, they were only able to take courses in their majors. The aim here was to allow students to specialize in selected fields (so no course outside the majors) but also avoid an overly narrow qualification (hence, the requirement to study three majors). Experience showed, however, that the level of the majors was often too low since three courses were not enough. At the same time, students lacked freedom to individually balance specialization in selected topics with a broader qualification covering more topics. Some changes were made and today's solution (see above) allows students to specialize in depth if necessary but also gives scope to gain a broader qualification. This option gives students responsibility for the decision to balance a very specialized qualification with a wider choice of topics.

Preparation for Master's thesis. In the first few years, it became apparent that many international students lacked sufficient experience and skills in writing a report that which meets the quality criteria for a scientific Master's thesis. This was due either to the lack of any written thesis in the Bachelor's degree or the style of the Bachelor's thesis, namely following the instructions of a supervisor and not working independently as required in a scientific project and expected at a German university. In order to overcome this problem, courses on scientific working and writing were introduced. Furthermore, instead of several short research projects prior to the Master's thesis, a longer research project was introduced equivalent to a Bachelor's thesis (300 hours) including a mandatory report. 
Supporting students. In an international program, the continuous support of students is vital as they have to deal with a new country, a new culture and a new university system. In the first few years, MAOT offered very individualized support, guiding students closely and personally through administrative issues such as acquiring a residence permit or issues of daily life. This was intended to offer the best possible support and also to gain extensive experience in supporting international students. Incrementally, the strategy changed to a mixture of more general support (by providing written guidelines and introductory events) with less individualized support. This turned out to be more time efficient and strengthened the independence and self-responsibility of the students. However, it was only possible on the basis of experience of numerous individual cases of student support. It also became feasible as soon as newly arrived students were able to receive reasonable support from senior students. In the first few years, there simply were not enough senior students to provide sufficient exchange between junior and senior students.

\section{Lessons learned}

The section above described developments in the program over the last ten years. In so doing, it included some lessons that had to be learned and which might be transferable to similar programs. However, the focus was on developments specific to MAOT and often refer to the curriculum. In this section, we summarize lessons learned that we consider potentially beneficial for similar programs or initiatives.

\section{Interdisciplinary}

Increasingly, interdisciplinarity became the defining characteristic - and main challenge - of the program. This came as a surprise because the international rather than interdisciplinary aspect on an international program may at first seem the most characteristic feature. In fact, using English as a working language (with almost no native speakers among the students and the lecturers) has a positive impact on everyday interaction (see below). However, interdisciplinary encounters between people (students and lecturers) from different backgrounds have turned out to be much more challenging.

Lecturers had to learn to adapt their lectures and also their supervision in research projects to a group of students much more heterogeneous than they are used to. Heterogeneous groups are not new to lecturers since they always teach students from other departments outside their home subject area. Usually, however, there is a clear differentiation, between when a lecturer is 'at home' (i.e. in their department) and when 'foreign' students are 'at home' (i.e. in the their department). This clarifies the roles in the interaction in terms of what prior knowledge is expected and who is supposed to possess the competence. This makes the interaction easier. Students have to adapt to courses where the lecturer is on his/her 'home turf' and benefit from his/her experience. The lecturer knows what he/she can expect from students and not rely so much on the students' own expertise.

In MAOT, however, students do not have a home department but rather work entirely interdisciplinary. They come from very varied backgrounds and are based at different departments currently across two faculties (intensive involvement of the medical faculty is rare and is therefore not considered as standard). Optics, the core competence, is 'at home' in the physics department rather than the faculty of engineering. However, their degree program is located in the faculty of engineering.

Lecturers have to learn to deal with this situation and understand which skills MAOT students can contribute - from their core competence in optics and from their experience of interdisciplinary work. By contrast, students have to learn to deal with the inconvenience of not having a stable 'home department' and often feel insecure about the own skills. They need to develop confidence in their own abilities, develop self-esteem, and find a way to contribute their knowledge to discussions and research projects.

Two illustrative examples might help:

- Courses and research projects in Computational Optics require good programming and mathematical skills, which MAOT students have to a lesser extent than students or lecturer from the Department of Computer Science. By contrast, MAOT students know much more about the physical processes to which the mathematical methods and the software are applied. In daily work, good programming skills are often the focus and may be a reason that MAOT students become marginalized. They need to learn to demonstrate their greater knowledge of physical processes and contribute this to the collaboration to benefit both sides.

- In the field of Optical Metrology, students require knowledge of the objects they are measuring and their properties, for example gas molecules or thermodynamic processes taking place within these gases. MAOT students often do not have this knowledge to the same extent as other students, for example from the field of 
chemical engineering. This lack is then criticized by supervisors or other students during lab courses or research projects. By contrast, MAOT students generally know much more about optical fibers, lenses or mirrors than other students of engineering. They can, therefore, contribute ideas to improving the optical setup in an ongoing measurement that outweighs their lack of knowledge about the objects under investigation. The actual exchange of knowledge and skills, however, is difficult. Both sides need to develop a shared understanding and the patience to deal with the need to combine strengths.

Issues arising from working across disciplines are not unique to MAOT but are present in almost all interdisciplinary academic contexts. They are not new to anybody working in science and research. The level of interdisciplinarity at MAOT, however, goes far beyond the level generally found in education and research and, consequently, the 'usual' issues occur to a much larger extent. While problems are more difficult to solve, the potential to develop skills in interdisciplinary work is equally higher. Also, the fact that MAOT is an interdisciplinary program in its very nature without the option to 'escape' to any 'home' department forces interdisciplinary collaboration.

The extensive interdisciplinarity also makes the issue of balancing a specialized education with a broad education more problematic. A highly interdisciplinary program offers students increasingly divergent academic directions. This might result in a curriculum that tends to be unbalanced (too narrow or too broad/ unspecific). This risk must be constantly kept in mind by the MAOT management, although experience tells us that it is best to rely on students' own responsibility (see below).

\section{International}

Language skills. It is often said that 'bad English' is the universal language of science; this also applies to international study programs in English, especially when offered in a non-English speaking country. However, students on programs in English-speaking countries may lack the language skills necessary to interact effectively with native speakers. The language issue, therefore, must not be underestimated. The interaction among students and between lecturers is constantly shaped by the existing command of English, impacting on time efficiency and quality of interaction. This needs to be balanced out with appropriate measures such as investing more time in interactions or learning to express oneself with limited capabilities.

It must be stated that the language skills of both lecturers and students do not necessarily improve by teaching or studying on an English-speaking program. Daily routine reinforces the existing level of competence and make people more confident in using the language, but it does not necessarily help improve skills by reducing mistakes, expanding vocabulary (apart from newly learned specialist terms), improving grammar skills or learning new phrases.

Also, there is often not much motivation to improve language skills. Those who may need to improve their English tend not to be aware. Moreover, other demands and constraints of study and research (in particular lab duties) often do not leave space even if they were aware and motivated.

Lecturers in international programs should be honest about their own language skills and consider the consequences for students' ability to learn from courses. Lecturers should either be required to improve their language skills or take measures to compensate for poor language skills.

Students in international programs often have to work on their language skills. It is vital that they understand the importance and the fact that a successful career in a research-oriented environment sometimes means prioritizing language learning at the cost of technical education.

Occasionally, students prepare for exams in their mother tongue, especially those students who feel less confident about English. This is more likely if a group of students come from the same country. In most cases, this strategy leads to serious problems in exams when knowledge must be produced in English and the students lack the appropriate vocabulary, or lack the time and energy to phrase answers in English. When managing international programs, it is important to make students aware of this risk and try to avoid it by introducing countermeasures such as establishing mixed-language work and study groups.

A particular aspect connected to language is that all parties should be honest and politely open with each other. Many communication problems occur from specific regional accents or other idiosyncrasies of using English as a foreign language. In limited interactions such as at conferences or the occasional meeting, it might not be possible to talk about the language issue frankly and honestly. In daily interactions on a study program the potential negative impact is so significant that an honest approach is necessary, even if uncomfortable. Experience at MAOT tells us that the situation can be improved if such honest conversations take place. 
Cultural aspects. Even more so than language problems, differences in working cultures, in ways of working and collaborating, and attitudes toward work impact daily collaboration in the classroom and the lab considerably. This concerns, for example:

- the importance attached to work in a person's value system (and consequently how much time he/ she is willing to dedicate to it)

- the level of independence and responsibility a person adopts for him-/ herself and, in this context, how much risk of failure he/ she is prepared to accept

- the understanding of how to work with authorities and how to interact between levels of hierarchy

- the quality standards a person sets for him-/ herself

- punctuality and the understanding of deadlines

- the awareness that students are responsible themselves for the organization and success of their study in many respects

All these aspects can lead to serious problems for joint working in the classroom or the lab. The general discussion on how to approach this 'clash of cultures' is hotly debated with a range of attitudes toward it. The MAOT experience has shown that the basic strategy is to accept the culture of the host country as the basis for collaboration - to a larger extent where research projects are concerned, to a lesser extent for interaction in the classroom. In the case of MAOT, it is the German academic culture and, therefore, people from other countries must adapt.

However, this process must happen in a way that is acceptable to those who are part of it. These main aspects should be considered:

- The representatives of the host country should consider the extent to which the standards of their culture need to be adopted. They should not follow their very personal views or make the mistake of using a stereotypical embodiment of the cultural standard (which tends to be much stricter than the actual one). Usually, the application of standards differs remarkably within a culture itself; it might be wise to set the demands for international people at a lower level, which may not represent the average standard in the host country but which is still acceptable.

- Standards should not be just set, but explained. Often, a single aspect such as how much independence a supervisor expects from a student is hard to understand on its own, but easier if it is set in the context of a concept for the whole working process. Supervisors and colleagues should take the time to provide these explanations, which might often require more reflection than usual on personal habits.

- International students should have enough time to adapt. It is not enough to explain standards once and then expect that these will be adapted quickly. This process takes a certain amount of time to really succeed, even up to a year.

\section{Individual}

Providing a great deal of freedom to the students proved to be a good strategy and enabled them to follow individual paths. MAOT adopted this strategy from the very beginning and, after initial doubts as to how students would handle this degree of autonomy, developed it further. In fact, these doubts were justified as the students did actually have problems with this responsibility (see above). In particular, the freedom to choose a highly individualized curriculum sometimes led to students choosing courses that did not fit together. This resulted in a lack of connection and synergy between courses.

The solution, however, is not to reduce the degree of freedom and individuality, but rather to support students better in finding their individual path. This is best achieved by combining an open attitude that respects individual student decisions (within the constraints of the exam regulations and other regulations of the university) with regular open and honest feedback. Sometimes this is offered voluntarily and sometimes asked for specifically. Feedback should be given on a professional basis using solid justifications. Lectures and other support staff can and should be free to share their personal perspectives, but should separate personal opinion from professional assessment. In extreme cases, a student's choice of curriculum may be considered wrong, but this can be discussed in a respectful manner and with valid justification. 
Another way to support the individual choices of students is general guidelines that cover key issues affecting student decisions and provide a comprehensive analysis from all perspectives. This can be in the form of student guides, information events or regular messages to students (via mailing lists or bulletin boards) at the time a decision is due. Direct messages to students are particularly effective as these can be scheduled to arrive when a decision is due (information from general guides or an information event are sometimes forgotten by the time it is required). This may also seem be less formal and, therefore, seem less important to students when making their own decision. For this method of 'in-time' messages to succeed, the messages need to be include a balanced analysis of or recommendation for a decision.

Naturally, it cannot be guaranteed that freedom for students will always result in them taking the right decisions (since 'right' is always subjective). Experience at MAOT, however, shows that students - if well supported - are generally able to take decisions that lead to a good outcome, even if not the best outcome. Initially, many students may have difficulties in knowing how to make good decisions. They may hesitate and feel insecure, change their mind several times, regret decisions, and sometimes find that they have to take a step back and pursue another path. It is important then to help them understand that this is all part of the process of developing personal skills. It is also crucial to give them the time to develop these skills and to empower them to allow themselves this time. Students of technical subjects especially tend to want to develop personal skills as fast as learning from a textbook and using similar approaches (reading or listening to something, understanding and then mastering it). However, the development of these skills requires a sometimes slow process. The fact that they need to adapt to a new cultural environment at the same time - which always also involves developing new personal skills - make the situation even more difficult.

Our experience at MAOT has shown that most students are able to grow to deal with the challenges and the freedom given to them as long as they have a welcoming and supportive environment. There is probably no other possibility anyway on an interdisciplinary and international program. A programe such as MAOT accepts students from very different backgrounds (in terms of the country and the subject area they come from). This means that they have all decided to follow a very individual path, otherwise they would have rather stayed in their home country or with their previous subject. A program such as MAOT, therefore, deals with distinctive personalities who attach great value to their individuality. If the program's strategy tried to work against this, it would be in such severe contradiction to the personalities of its students that constant battles between program and students would arise, hindering successful learning to an unacceptable extent.

Unfortunately, however, in some cases the chosen strategy will still not work due to the cultural impact or problems arising from a student's personal situation (for example mental health or family issues) that are too big to be solved with the support of a Master's program. Then, the program management might suggest that a student leave the program earlier, but these are very rare exceptions.

\section{Conclusions}

Interdisciplinary, international and individualized Master's programs are possible and are a feasible way of addressing the lack of education in the field of optics and optical technologies. However, they cannot be devised and run like a standard Master's program at a university; the particular aspects described in this article need to be considered. Also, each new program will take several years to become fully established. Changes may need to be made to the strategy and procedures to ensure success and meet the standards set.

\section{REFERENCES}

[1] https://www.elitenetzwerk.bayern.de/elite-network-home/elite-network-home/?L=2

[2] Bundeministerium für Bildung und Forschung: Photonik Forschung Deutschland, 2011, https://www.bmbf.de/pub/Photonikforschung_in_Deutschland.pdf (29.03.2017)

\section{ACKNOWLEDGEMENTS}

The authors gratefully acknowledge funding from the Elite Network of Bavaria and the support of the Erlangen Graduate School in Advanced Optical Technologies (SAOT). 